\title{
Gemeinsame Entscheidungen sind besser
}

\begin{abstract}
Ärzte, die an einer Schulung über gemeinsame Entscheidungsfindung teilgenommen haben, verordnen bei Infekten der oberen Luftwege signifikant weniger Antibiotika, ohne dass die Patienten deswegen unzufriedener mit der Behandlung sind.
\end{abstract}

- Die Auswertung der Daten einer großen deutschen Krankenkasse zeigte erst dieser Tage, dass der Anteil von Kindern, die unter der Diagnose Mittelohrentzündung Antibiotika verordnet bekommen haben, in den letzten zwei Jahren um 26\% gestiegen sei.

Von vielen Ärzten ist zu hören, dass die Patienten selbst die treibende Kraft für diese Entwicklung sind, da sie vom Arzt für sich oder ihre Kinder Antibiotika fordern. Eine randomisierte Studie aus der kanadischen Provinz Quebec zeigt, dass diese Entwicklung durch mehr Information umkehrbar ist. Allgemeinärzte aus der Studiengruppe nahmen an einem zweistündigen OnlineTutorial über die Symptomatik akuter respiratorischer Infekte, die Wirkung von Antibiotika, einer effektiven Kommunikation mit dem Patienten und einer Beteiligung des Patienten an der therapeutischen Entscheidung teil.

Bei den derart geschulten Ärzten bestanden nur 27,2\% auf eine Antibiotikaverordnung bei Mittelohrentzündung, in Kontrollpraxen waren es $52,2 \%$. Wie $\mathrm{zu}$ erwarten, bestand zwei Wochen nach Beendigung der Behandlung kein Unterschied im klinischen Ergebnis. Allerdings waren die Patienten, die in die Therapieentscheidungen mit einbezogen wurden, mit dem Ablauf der Behandlung signifikant zufriedener.

\section{Kommentar}

Man darf annehmen, dass die Patienten der Studiengruppe mehr Zeit für das Gespräch mit dem Arzt in Anspruch nahmen. Und genau dieser Umstand dürfte der wesentliche Faktor für die größere Zufriedenheit mit der Behandlung sein, blieb doch der klinische Verlauf unbeeinflusst. Diese Zeit bekommt ein deutscher Arzt nicht honoriert. Wenn die Kassen also über zu häufige Antibiotikaverordnungen klagen, sollten sie auch den erhöhten Zeitaufwand des Arztes honorieren, der erforderlich ist, um einen Patienten zum Antibiotikaverzicht zu überreden.

H. S. FÜESSL =

- F. Légaré et al.

Training family physicians in shared decisionmaking to reduce the overuse of antibiotics in acute respiratory infections: a cluster randomized trial. Can. Med. Assoc. J. 2012; doi: 10.1503/cmja.120568

\section{Quelle im Magen}

Ein Mann wurde wegen akuter Hämoptysen als einziges Symptom ins Krankenhaus aufgenommen. Er war bisher nicht krank gewesen und stand nicht unter Medikamenten. Außer einer leichten Erhöhung der Transaminasen und einer normozytären Anämie waren die Laborbefunde unauffällig.

- In der Computertomografie des Thorax erkannte man eine kleine ovaläre Verschattung im Bereich des Mittellappens. Weitere Rundherde fanden sich in der Leber und der Milz, sodass der Verdacht auf eine Metastasierung eines bislang nicht bekannten Karzinoms geäußert wurde. Eine gründliche dermatologische Inspektion der Haut ergab keinen Befund, der mit einem malignen Melanom oder einem anderen Malignom der Haut vereinbar gewesen wäre. Auch die Bronchoskopie ergab weder die Blutungsquelle noch

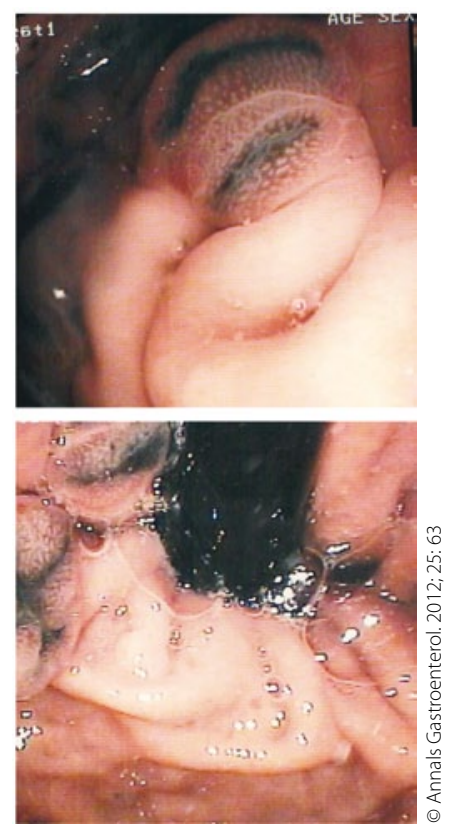

Melanom im Magen und Duodenum.

einen hinweisenden pathologischen Befund.

Im Zuge der weiteren Abklärung wurde auch eine Gastroskopie durch- geführt, bei der sich ein ausgedehnter, schwarzbraun gefärbter Tumor fand, der fast den ganzen Magen auskleidete. Auch im Duodenum waren schwarzbraune Läsionen zu sehen. Die Koloskopie war unauffällig. Histologisch erwies sich der Befund im Magen als invasives gastroduodenales malignes Melanom mit positivem immunhistochemischen Befund für Protein S-100 und HMB-45.

\section{Kommentar}

Melanome des Gastrointestinaltrakts sind sehr selten, haben hohes malignes Potenzial und eine schlechte Prognose. Wegen der frühzeitigen Neigung zur Metastasierung werden sie in der Regel über Komplikationen der Metastasen entdeckt.

H. S. FÜESSL =

- N. V. Chysanthos et al.

(General Hospital of Chania, Greece; Korres.: nchrysanthos@yahoo.gr): Gastroduodenal melanoma in a male patient with hemoptysis. Annals Gastroenterol. 2012; 25: 63 